\title{
Reflexiones y criterios relativos a la creación de modelos BIM de edificios históricos
}

\section{Reflections and criteria related to the creation of BIM models of historical buildings}

\author{
Rafael Martín Talaverano ${ }^{1}$ \\ Escuela Técnica Superior de Arquitectura, Universidad Politécnica de Madrid \\ José Ignacio Murillo Fragero² \\ Urbe pro Orbe Patrimonio Cultural, S.L. \\ María de los Ángeles Utrero Agudo ${ }^{3}$ \\ Escuela de Estudios Árabes, CSIC
}

\section{RESUMEN}

El presente artículo tiene como objetivo fundamental analizar el proceso de creación del modelo BIM aplicado al ámbito de los edificios históricos, considerando las singularidades de los mismos para lograr un uso adecuado y eficaz. Para ello, se han estudiado tres casos de construcciones pertenecientes al conjunto de la arquitectura altomedieval hispana, en los cuales se han desarrollado distintas estrategias de modelado para analizar aspectos como el nivel de detalle o el de precisión métrica, así como el nivel de desarrollo (LOD) adaptado a las particularidades de la arquitectura histórica. Como resultado de esta investigación, se expone una serie de conclusiones que giran en torno a las claves para alcanzar la viabilidad de la aplicación de la metodología BIM en el ámbito del patrimonio cultural, tanto en la fase de creación del modelo como en relación con el uso del mismo.

Palabras clave: HBIM; patrimonio cultural; patrimonio construido; secuencia evolutiva; arqueología de la arquitectura; arquitectura altomedieval.

\begin{abstract}
The main objective of this paper is to analyse the creation process of a BIM model applied to the field of historical buildings, taking into consideration their specific features in order to reach a successful and efficient use. For this purpose, three early medieval buildings have been studied. A specific modelling strategy has been applied to each of them and the research has been focused on several aspects, such as the level of formal simplification, the level of metric accuracy or even the level of development (LOD) properly adapted to the singularities of historical buildings. As result of this analysis, some conclusions are described, of which common thread is to propose keys to reach the feasibility of the application of the BIM methodology in the field of cultural heritage, both during the creation of the model and when using it.
\end{abstract}

Key words: HBIM; cultural heritage; built heritage; evolutionary sequence; archaeology of architecture; early medieval architecture.

Recibido: 03-02-2020. Aceptado: 01-04-2020. Publicado: 08-04-2021

Cómo citar este artículo / Citation

Martín Talaverano, R., Murillo Fragero, J. I. y Utrero Agudo, M.a Á. 2021: "Reflexiones y criterios relativos a la creación de modelos BIM de edificios históricos", Arqueología de la Arquitectura, 18: e113. https://doi.org/10.3989/arq.arqt.2021.005

Copyright: (C) CSIC, 2021. (C) UPV/EHU Press, 2021. Este es un artículo de acceso abierto distribuido bajo los términos de la licencia de uso y distribución Creative Commons Reconocimiento 4.0 Internacional (CC BY 4.0). 


\section{INTRODUCCIÓN}

\subsection{BIM y patrimonio cultural}

El empleo de la metodología BIM (Building Information Modeling) ha supuesto en los últimos años un gran avance en el sector de la arquitectura, la ingeniería y la construcción (Architecture, Engineering and Construction, AEC). Su objetivo es potenciar el empleo de un modelo de información unificado y completo del edificio sobre el cual desarrollar los distintos trabajos de un modo coordinado, sistemático y eficiente por parte de todos los agentes que intervienen en él. En el marco de la obra nueva, entre otros aspectos, se mejora el diseño y la definición del proyecto, evitando costes derivados de la aparición de contradicciones o interferencias. De este modo, la inversión de la implantación de la metodología BIM genera beneficios que apoyan su viabilidad.

Estos beneficios han animado el análisis de la aplicación del entorno BIM en el marco del patrimonio cultural, derivándose de ella el concepto de HBIM (Murphy, McGovern y Pavia 2009). Son variadas las revisiones sobre el tema realizadas hasta la fecha, centradas en el modelado y la implantación en plataformas BIM (Pocobelli et al. 2018), en la tecnología y los componentes BIM (Logothetis, Delinasiou y Stylianidis 2015), en los distintos enfoques metodológicos y las herramientas (López et al. 2018), en las dimensiones y niveles de madurez (Merchán et al. 2018), en los usos y los protocolos (Jordan et al. 2018; Salvador, García-Valldecabres y Viñals 2018), así como en los gemelos digitales y la conservación preventiva (Jouan y Hallot 2020). Sin embargo, no se ha reflexionado sobre la creación del modelo HBIM partiendo de las características específicas y valores propios de la arquitectura histórica, vacío que pretende abordar este trabajo.

\subsection{Características de la arquitectura histórica}

Los edificios históricos tienen características que los diferencian de los contemporáneos, destacando dos de ellas por su relevancia en relación con la adaptación de la metodología BIM: la configuración constructivo-material y la dimensión temporal.

Respecto a la configuración constructiva-material de los edificios históricos, se debe señalar que estos emplean diferentes elementos y técnicas constructivas pro- pias de los sucesivos periodos en los que se concibieron y modificaron. Aunque podamos encontrar materiales comunes en la arquitectura contemporánea, las técnicas y tecnologías difieren. Pero la diferencia no reside únicamente en el material, sino en el modo en el que este se emplea, las herramientas con las que se trabaja y el conocimiento que poseen los artífices de estas obras arquitectónicas. La ausencia de producción estandarizada explica por qué no hay dos edificios iguales, ni dos elementos idénticos.

Esta configuración es consecuencia de una secuencia histórica. Las construcciones no son el resultado de una única acción, sino de un conjunto de acciones constructivas y destructivas que se suceden a lo largo de su historia y que dejan su huella material, caracterizándose cada una de ellas por técnicas y tecnologías distintas, como hemos indicado arriba. El edificio histórico comprende así una serie de construcciones, espacios y usos que han convivido o no durante su vida útil. Mientras tenga uso, la obra será objeto de cambios, prolongándose su secuencia. Por el contrario, el edificio actual prácticamente carece de esa secuencia, ya que es resultado de un proyecto unitario y reciente. Una vez comience su uso, a menudo materializado en transformaciones, se convertirá en un edificio histórico.

\subsection{Contexto, objetivos y método de la investigación}

La aplicación de la metodología BIM al ámbito del patrimonio cultural se ha desarrollado, hasta la fecha, en varias líneas. Como señalan algunos autores (Castellano y Pinto 2019), algunas investigaciones se han centrado en el modelado de geometrías complejas propias de este tipo de edificios; otras se han dirigido a la búsqueda de una máxima precisión en modelos BIM a partir de levantamientos 3D; y otras se han ocupado del empleo de BIM para la generación de conocimiento del propio edificio histórico. En esta última dirección, algunas investigaciones (Bruno y Roncella 2019) han propuesto el diseño de un sistema de información enfocado a la implementación de datos no geométricos y a su empleo en el mantenimiento y la conservación del patrimonio construido. Pero estas propuestas siguen focalizando su atención en las herramientas tecnológicas o en las intervenciones sobre el patrimonio, dejando completamente al margen los valores descritos de la arquitectura histórica como base fundamental para la organización del modelo HBIM y del sistema de información. Estos enfoques 
no permiten abordar en consecuencia un aspecto crucial para la implementación de la metodología BIM, como es su viabilidad dentro de un sector, el del patrimonio cultural, relativamente frágil y escaso de recursos.

Por todo ello, asumiendo como variables fundamentales las características específicas de la arquitectura histórica, hemos intentado formular conceptos de apoyo prácticos para la creación de modelos HBIM que ofrezcan una definición más ajustada a las necesidades del patrimonio histórico, incorporando a la reflexión la viabilidad, aspecto clave para que su implantación ofrezca un beneficio al sector. Para ello, se han seleccionado tres casos de estudio pertenecientes a un periodo arquitectónico común y que han sido analizados desde la metodología de la Arqueología de la Arquitectura. Se trata de las iglesias altomedievales de Santiago de Peñalba (Peñalba de Santiago, León), San Pedro de la Mata (Sonseca, Toledo) y San Cebrián de Mazote (San Cebrián de Mazote, Valladolid). Los tres edificios cuentan con una completa documentación gráfica, obtenida mediante diversas técnicas de levantamiento: en el primer caso, se disponía de una planimetría completa (dibujos 2D) como base para el modelado; en el segundo, de un modelo fotogramétrico estereoscópico de líneas 3D, las cuales han servido de apoyo al modelado; y en el tercero, se contaba con modelos 3D de nube de puntos y de mallas obtenidos con fotogrametría convergente. Queremos subrayar que no pretendemos realizar un análisis sobre las técnicas o las herramientas de modelado, sino una reflexión sobre los criterios para conseguir una mejor adaptación de la metodología BIM al ámbito del patrimonio cultural. La variedad de los datos gráficos de partida nos ha permitido contrastar distintos criterios y resultados.

\section{MODELADO HBIM DE EDIFICIOS HIS- TÓRICOS}

El primer paso hacia la creación de un modelo HBIM debe ser la planificación inicial. Aunque esta fase no es el objetivo de este trabajo, conviene recordar que esta debe considerar una gran variedad de aspectos, tales como el tipo de bien cultural y el nivel de conocimiento del mismo, sus valores y características, su documentación asociada, su estado de conservación, sus usos presentes y futuros o su marco de gestión (una descripción más detallada de estos aspectos en Guía de Usuarios BIM 2018). Independientemente de la orientación, contenidos y usos definidos para el modelo HBIM, este siempre se sustenta en dos pilares fundamentales: un sistema de información asociado a un modelo geométrico del edificio.

\subsection{Sistema de información HBIM}

Dado que uno de los objetivos fundamentales del empleo de la metodología BIM es el de mejorar la gestión de los datos, el sistema de información debe adecuarse al nivel de conocimiento y a las características del edificio histórico. El concepto de nivel de conocimiento ha sido abordado por diferentes autores. Algunos hacen referencia al "Level of Knowledge" definido en los "Technical Standards for Construction" en Italia (Bruno y Roncella 2018: 177), si bien se limitan a establecer en qué medida se conocen los aspectos geométricos, constructivos y estructurales. Otros autores proponen sin embargo nuevas aproximaciones, aunque más ligadas a los usos del modelo BIM en relación con la tutela del patrimonio cultural (Castellano y Pinto 2019). De un modo análogo, se ha propuesto el "Grade of Information" también vinculado a los distintos tipos de usos del modelo HBIM (Banfi 2017: 61).

Sin embargo, entendemos que el nivel de conocimiento de los edificios históricos va más allá de lo anteriormente citado, ya que cubre un gran abanico de aspectos relativos al propio edificio y a sus contextos histórico, tecnológico, cultural, social, etc. En relación con la metodología HBIM, proponemos como aspecto fundamental una de las singularidades de la arquitectura histórica antes referida: la secuencia temporal. El sistema de información del modelo HBIM debería, en nuestra opinión, articularse en torno a ella, convirtiéndose esta en su eje vertebrador, ya que dicha secuencia se ajusta con rigor a la naturaleza del edificio histórico y permite vincular los datos de un modo coherente y lógico. Su identificación es posible gracias a la aplicación de la metodología de la Arqueología de la Arquitectura (Caballero 2010), la cual determina rigurosamente las distintas fases y transformaciones que configuran la realidad evolutiva del edificio.

En relación con los casos de estudio desarrollados en el marco del presente trabajo, el sistema de información se ha organizado por ello en torno a la secuencia histórico-constructiva obtenida gracias a la metodología arqueológica, representada gráficamente en el diagrama de relaciones estratigráficas periodizadas (Fig. 1). El diagrama, además de sintetizar la secuencia, permite estructurar la base de datos, la cual se ha implementado en el correspondiente entorno BIM mediante una aplicación 

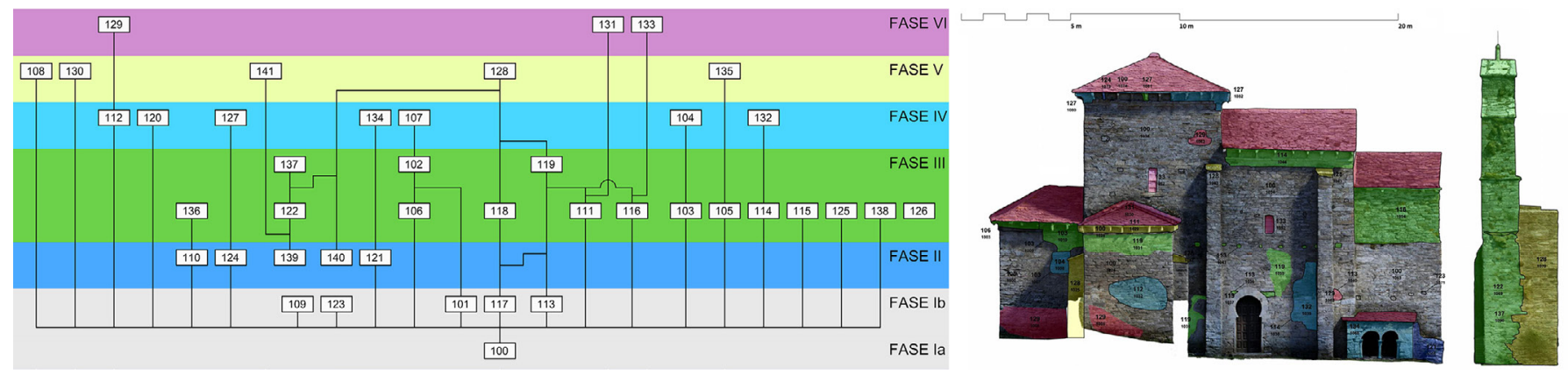

Figura 1. Iglesia de Santiago de Peñalba. Diagrama estratigráfico y alzado norte. Fuente: elaboración propia.

informática especialmente diseñada para el ámbito del patrimonio cultural.

Una vez estructurado de un modo coherente el sistema de información con la naturaleza del edificio histórico, debe ser capaz de incluir el tipo de datos propio de este ámbito (esto es, datos históricos, artísticos, arqueológicos, constructivos, estructurales, patológicos, etc.). Sea cual sea la herramienta BIM que se emplee, esta debe poder configurarse de tal modo que el usuario tenga la opción de implementar este tipo de datos adecuadamente.

\subsection{Generación del modelo geométrico}

La creación del modelo geométrico debe tener en cuenta en todo momento los requisitos y objetivos iniciales para poder ajustarse a un marco de viabilidad adecuado. En este sentido, es importante atender a dos aspectos.

Por un lado, el nivel de detalle establece en qué medida el modelo se acerca a la apariencia real del objeto (no debe confundirse con el nivel de desarrollo, cuyas siglas son iguales). Por ejemplo, una columna puede modelarse con mucho detalle (incluyendo todos sus ornamentos) o, por el contrario, de un modo simplificado y conceptual (incluso con elementos geométricos alejados de su apariencia real). Este aspecto es clave de cara a la viabilidad del modelo, ya que un nivel de detalle alto requiere mayor tiempo de modelado. En cualquier caso, suele ser necesario asumir algún tipo de simplificación de la geometría, entendiendo además que no es el objetivo principal del modelo HBIM aportar una representación realista. No obstante, es difícil establecer un parámetro numérico para definir este aspecto.

Por otro lado, el nivel de precisión métrica establece la desviación máxima admisible respecto a la geometría del edificio y, aunque está relacionado con la apariencia más o menos realista establecida en el nivel de detalle, es un concepto distinto. Algunos autores (Banfi 2017: 62; Bruno y Roncella 2018: 177) hacen referencia a este concepto denominado como "Level of Accuracy" (LOA) o "Grade of Accuracy" (GoA). Al contrario que en el caso anterior, este aspecto es fácilmente analizable gracias a la funcionalidad que ofrecen varias aplicaciones de gestión de nubes de puntos, las cuales permiten detectar las diferencias métricas entre el modelo y la realidad del objeto (Banfi 2017: 62).

Las diferentes estrategias de modelado tendrán que adecuarse a los aspectos citados para cumplir los objetivos iniciales. Dichas estrategias están ya analizadas y clasificadas en lo que se ha denominado "Grade of Generation" (Banfi 2017: 60-61). Se pueden sintetizar del siguiente modo:

a. Creación de nuevos elementos paramétricos con operaciones geométricas estándar (GOG1 a GOG8). Este es el caso empleado para el modelado de la iglesia de Santiago de Peñalba, cuyos elementos constructivos se han creado mediante prismas generados a partir de la planta y las secciones con operaciones de extrusión, unión y sustracción de volúmenes (Fig. 2). En términos generales, esta estrategia proporciona los resultados menos precisos, pero con un menor coste de tiempo de ejecución.

b. Empleo de líneas o puntos extraídos del modelo de levantamiento para crear elementos no estándar (GOG9 y GOG10). Es posible así generar elementos con formas irregulares o deformadas (bóvedas, muros desplomados, etc.), si bien el tiempo de ejecución es mayor. Este es el ejemplo de San Pedro de la Mata, donde se han creado cada una de las caras de los muros mediante superficies NURBS adaptadas a las líneas de contorno de los sillares del modelo de levantamiento obtenido con fotogrametría estereoscópica (Fig. 3). 


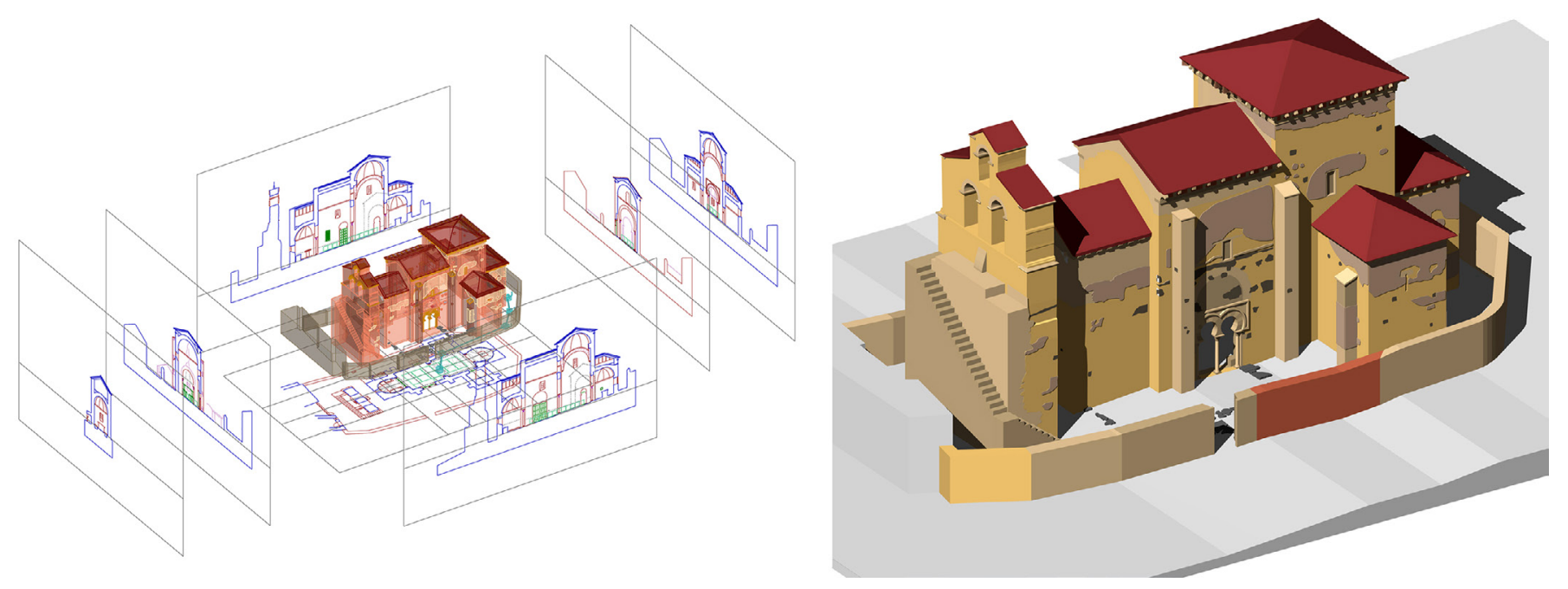

Figura 2. Modelo de la iglesia de Santiago de Peñalba. Fuente: elaboración propia.
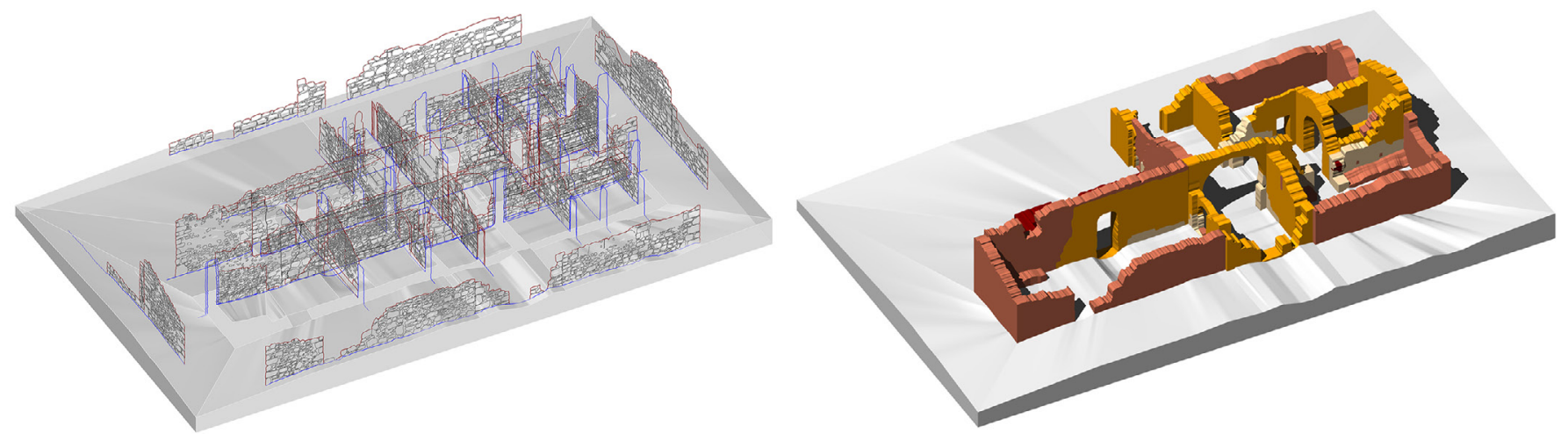

Figura 3. Modelo de la iglesia de San Pedro de la Mata. Fuente: elaboración propia.

c. Empleo directo del modelo de levantamiento (mallas), el cual es preciso dividir según los criterios adecuados. Mediante este sistema se obtienen elementos con formas muy complejas (capiteles, retablos, esculturas, etc.) y, por lo tanto, el mayor nivel de precisión y de detalle. Este enfoque no ha sido desarrollado hasta la fecha en otros estudios, debido probablemente a que su principal problema radica en la conversión de las mallas en elementos paramétricos manejables en entornos BIM. En el marco de este trabajo, se presenta esta novedad, ya que esta es la estrategia empleada para el modelado de la iglesia de San Cebrián de Mazote (Fig. 4; Martín, Murillo y Utrero 2020).

\subsection{Nivel de desarrollo del modelo HBIM}

Un aspecto fundamental a la hora de abordar la creación de un modelo BIM y su viabilidad es el nivel de desarrollo (LOD). En el caso de las obras de nueva

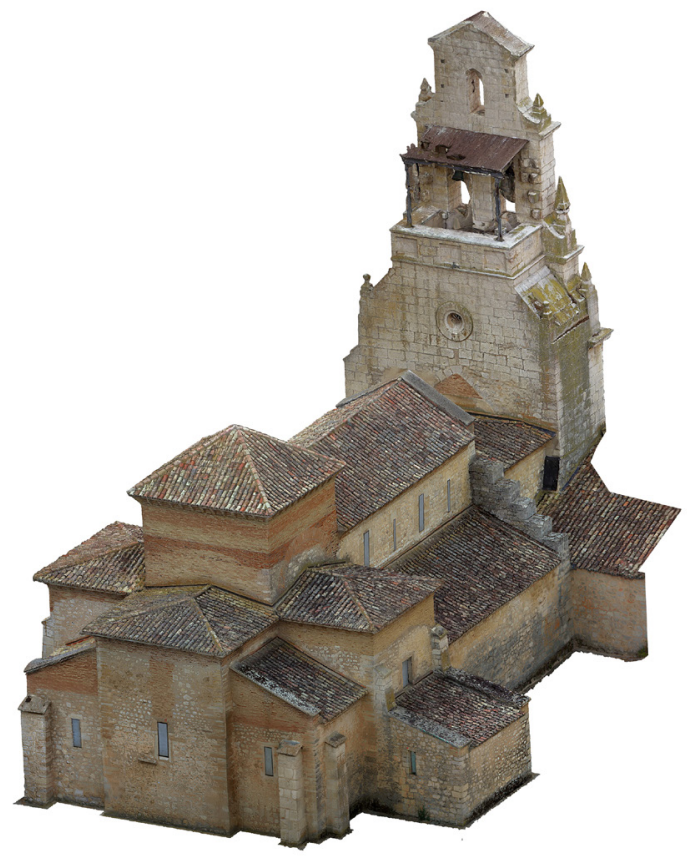

Figura 4. Modelo de la iglesia de San Cebrián de Mazote. Fuente: elaboración propia. 
planta, las características de cada nivel están bien definidas (The American Institute of Architects 2013). Sin embargo, en el ámbito del patrimonio cultural, la problemática es radicalmente distinta, tanto por las diferencias ya enunciadas entre la arquitectura histórica y la de nueva planta, como por los distintos procesos que se desarrollan en cada caso. Existe un debate sobre la adaptación de este aspecto desde la obra nueva al ámbito del patrimonio cultural, en el que algunos autores proponen como equivalente el denominado "Level of Knowledge" (Castellano y Pinto 2019), si bien entendemos que queda limitado al tipo de uso que va a tener el modelo HBIM. Por lo tanto, creemos que la definición de LOD para el patrimonio cultural debe ampliarse con la consideración de las propias características y valores patrimoniales.

En este sentido, además de los ya mencionados niveles de detalle y de precisión métrica, proponemos como aspecto crucial para la definición del LOD de un modelo HBIM la incorporación del nivel de división de dicho modelo. El nivel de división está estrechamente ligado con el nivel de conocimiento del edificio histórico y con la configuración del sistema de información, ya que el modelo deberá estar dividido en tantos objetos como sea necesario para poder vincular los datos de una manera rigurosa y clara. Por ello, es muy importante definir desde el principio qué tipo de información se va a implementar y a qué elementos afecta esta última.

Aunque es prácticamente imposible definir y sistematizar a priori toda la casuística de tipos de información, sí queremos destacar que la configuración constructiva y la secuencia histórica son dos aspectos cruciales para planificar la división del modelo. Como antes hemos explicado, el primer aspecto hace referencia a la componente material de los edificios $\mathrm{y}$, de un modo análogo al que proponen algunos autores (Bruno y Roncella 2019: 9), se pueden establecer cuatro categorías de división:

1. Modelo sin división alguna (elemento unitario).

2. División según cuerpos, estructuras o zonas principales.

3. División según elementos constructivos (muros, forjados, bóvedas, etc.).

4. División según materiales constructivos (sillares, mampuestos, vigas, etc.).

A esta componente material, se le debe añadir la temporal, es decir, la secuencia histórica, según la cual proponemos las cuatro categorías de división que ya establece la metodología de la Arqueología de la Arquitectura:

1. Modelo sin división alguna (elemento unitario).

2. División según las fases históricas.

\section{Edificio histórico}

A.- Valores patrimoniales: nivel de conocimiento y características singulares del edificio

B.- Planificación: objetivos, recursos y usos del modelo HBIM

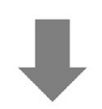

Modelo HBIM

A.- Sistema de información

B.- Modelo geométrico

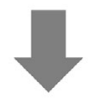

Nivel de desarrollo

Nivel de división

Nivel de simplificación formal

Nivel de precisión métrica

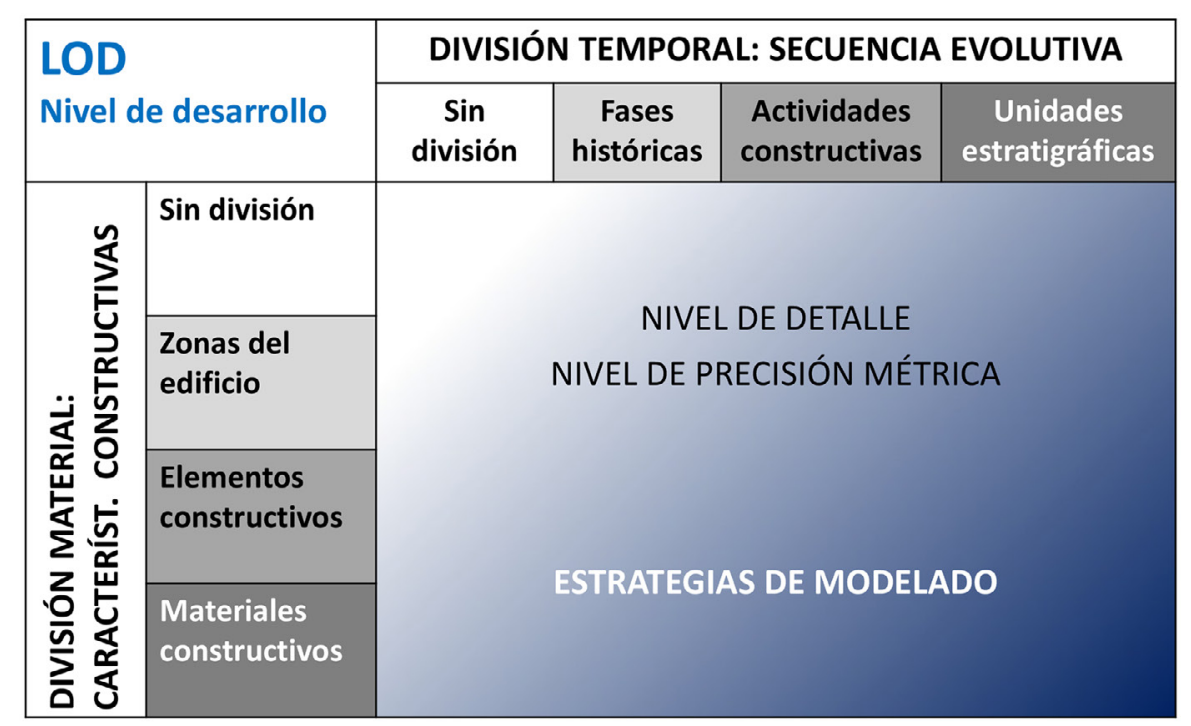

Figura 5. Esquema resumen de criterios de modelado HBIM. Fuente: elaboración propia. 


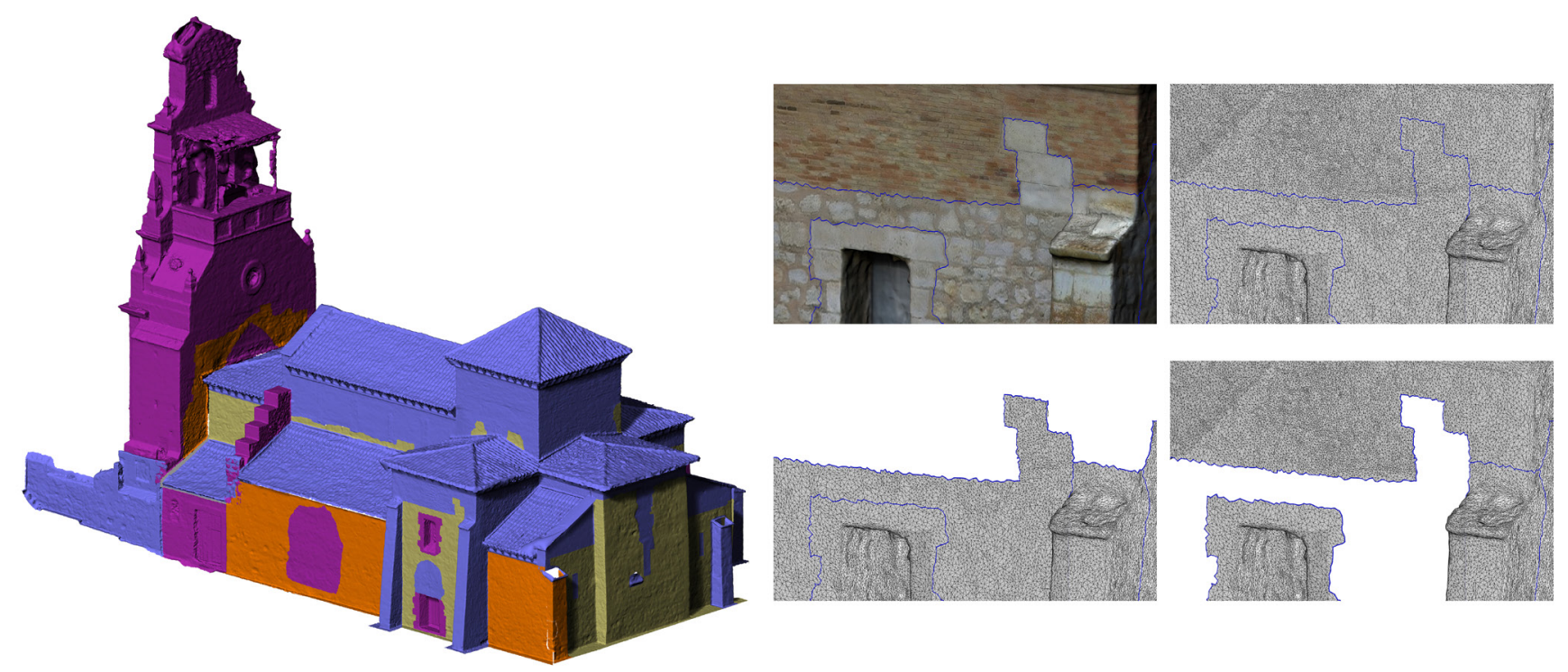

Figura 6. División del modelo de la iglesia de San Cebrián de Mazote. Fuente: elaboración propia.

3. División según las actividades constructivas o destructivas.

4. División según las unidades estratigráficas.

La combinación de estos dos parámetros (material y temporal) configura una matriz bidimensional en la que, junto con el nivel de detalle y el nivel de precisión métrica, se puede definir un LOD ajustado a la realidad del edificio histórico y adecuado para implementar un sistema de información con sus valores patrimoniales (Fig. 5). Complementariamente, debería considerarse el estado de conservación y la patología del edificio como criterios de subdivisión del modelo (Martín, Cámara y Murillo 2018), puesto que los procesos patológicos no tienen por qué afectar a elementos tal y como están definidos por su configuración constructiva o temporal (por ejemplo, una humedad puede afectar únicamente a una parte de un muro, que es una unidad constructiva). No obstante, este aspecto debe definirse en cada caso concreto.

En relación con los modelos generados en el marco de la presente investigación, los resultados del proceso de división han sido muy dispares en función de la estrategia de modelado. En el caso de San Cebrián de Mazote, donde se han empleado directamente las mallas del levantamiento, la división de las mismas ha derivado en un proceso muy complejo y lento, por lo que se ha optado por un nivel de división tipo 2 (Fig. 6). Sin embargo, en Santiago de Peñalba y San Pedro de la Mata, al realizarse en ambos casos el modelo con elementos geométricos paramétricos, su manejo y edición es más sencillo y rápido, por lo que se ha podido llegar a un

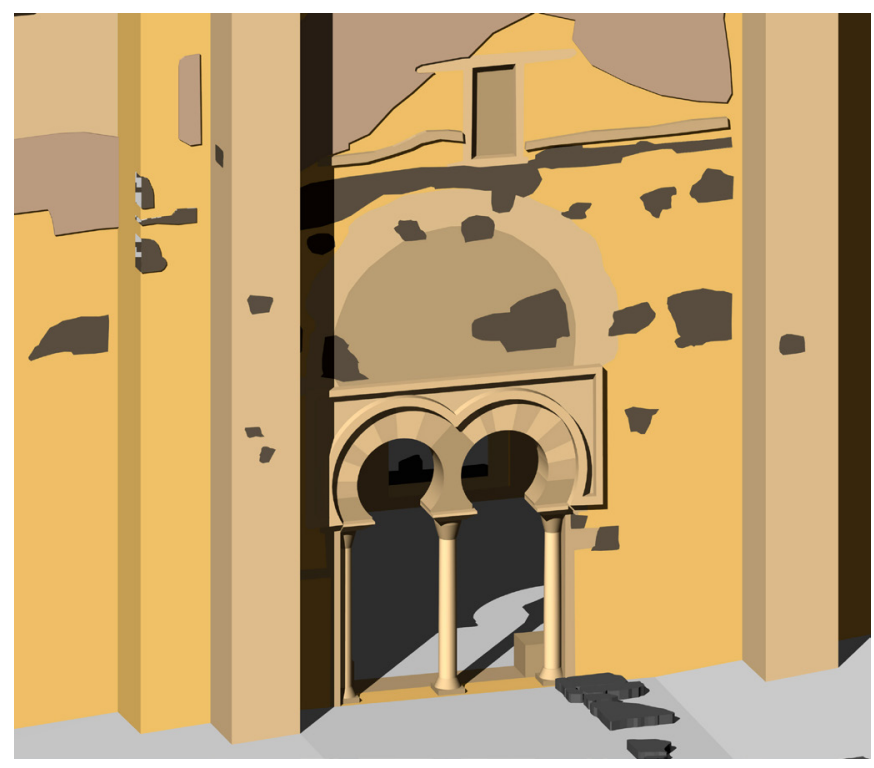

Figura 7. División del modelo de la iglesia de Santiago de Peñalba. Fuente: elaboración propia.

nivel de división tipo 3 en relación con la configuración constructiva y tipo 4 en relación con la secuencia temporal (Figs. 7 y 8 ).

A la vista de estas experiencias, podemos concluir que el empleo directo de las mallas obtenidas del levantamiento fotogramétrico no es recomendable por el momento, ya que su manejo y edición con las herramientas actuales son bastante laboriosos. No obstante, su utilización puede ser adecuada en elementos concretos de geometría muy irregular (por ejemplo, retablos, capiteles, etc.) que requieren un nivel de detalle muy alto. En 

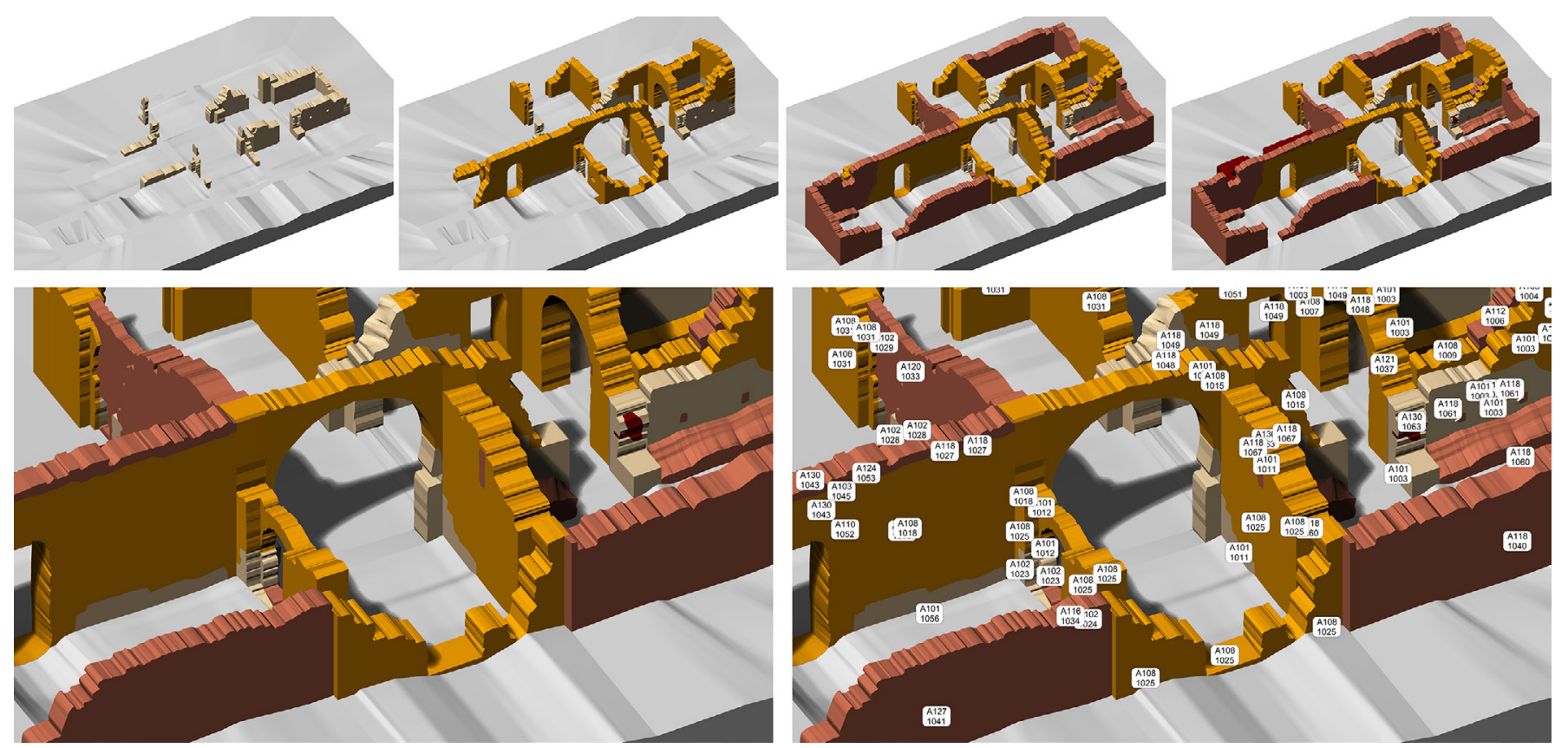

Figura 8. División del modelo de la iglesia de San Pedro de la Mata, tanto en fases históricas (fila superior), como en actividades y unidades estratigráficas (fila inferior). Fuente: elaboración propia.

cualquier caso, estos criterios deben planificarse inicialmente dentro de una estrategia global para la creación del modelo HBIM.

\subsection{Implementación de la información}

El trabajo desarrollado ha concluido con la implementación de la información sobre la secuencia histórico-constructiva en el modelo HBIM. A diferencia de otras experiencias dirigidas a este aspecto (Nieto y Moyano 2014), en la que los datos estratigráficos se asocian a nuevas entidades superpuestas a los elementos geométricos que configuran el modelo, en el presente trabajo toda la base de datos se organiza en base a la secuencia, de modo que los propios elementos del modelo HBIM tienen una iden- tificación temporal basada en el dato de su fase histórica, su actividad constructiva y/o su unidad estratigráfica. Este enfoque es completamente novedoso y ha sido posible gracias a la posibilidad de personalizar la base de datos que ofrece la aplicación informática empleada, algo fundamental para implementar un sistema de información acorde con la naturaleza del edificio histórico (Fig. 9).

\section{CONCLUSIONES Y REFLEXIONES FI- NALES}

\subsection{Sobre la creación del modelo HBIM}

La creación del modelo HBIM es un proceso que consume muchos recursos, por lo que debe ser cuidadosa-
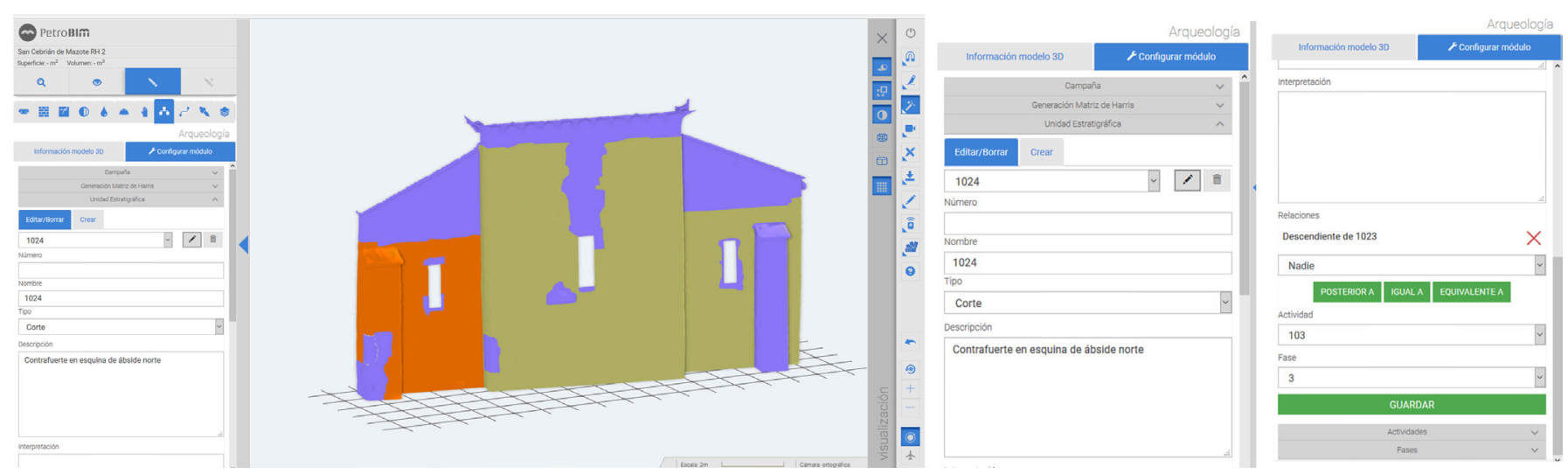

Figura 9. Implementación de la información estratigráfica en el modelo de la iglesia de San Cebrián de Mazote. Fuente: elaboración propia. 
mente planificado. Para ello, entendemos que es fundamental alcanzar una definición objetiva y rigurosa de los diferentes niveles de desarrollo (LOD), el cual debería integrar los conceptos de nivel de detalle, de precisión métrica y de división.

Entre los criterios expuestos en este artículo, conviene destacar el nivel de división, el cual está íntimamente ligado con la secuencia histórica del edificio, resultado de su análisis previo. Consideramos que esta secuencia debería ser el eje vertebrador del sistema de información del modelo HBIM, ya que es la estructura más coherente con la naturaleza del edificio para vincular el resto de los datos del sistema de información. Además, es preciso reflexionar sobre los requisitos relativos al nivel de detalle y de precisión métrica, ya que una elevada exigencia en estos aspectos conlleva un notable aumento de los recursos necesarios, los cuales no tienen por qué repercutir positivamente en la gestión de la información.

Finalmente, hay que considerar la configuración constructiva de los edificios históricos, caracterizados normalmente por la ausencia de sistematización y estandarización y por la presencia de soluciones particulares y específicas. El hecho de que el edificio histórico sea la materialización de una secuencia temporal implica además que sus elementos constructivos son imposibles de repetir, ya que cada uno de ellos es un tipo único y singular propio del momento en el que se realizó. Por ello, los intentos de generar bibliotecas o catálogos de elementos tipo que se puedan emplear de modo sistemático en distintos modelos HBIM son, en la mayoría de los casos, ineficaces y contrarios a la naturaleza evolutiva de estos edificios.

\subsection{Sobre el uso de la metodología BIM}

Como se ha comentado, la esencia de BIM consiste en la vinculación de un sistema de información a un modelo geométrico del edificio. Por ello, su principal ventaja es permitir una mejor gestión de los datos asociados. Sin embargo, a pesar de lo que varios autores defienden (Salvador, García-Valldecabres y Viñals 2018; Banfi, Brumana y Stanga 2019), creemos que HBIM no ofrece ventajas significativas en el ámbito de la difusión y la divulgación del patrimonio cultural hacia la sociedad, ya que no es una herramienta adaptada para la comunicación dirigida a un público general, sino para usuarios especializados. En este sentido, no debemos confundir el valor que los modelos 3D sí tienen en la difusión de los bienes culturales, gracias a su capacidad de potenciar la visualización y el entendimiento formal de dichos objetos por parte de usuarios no especialistas, con el valor del modelo HBIM. Este no es únicamente un modelo 3D y, en muchas ocasiones, está simplificado, es menos realista y visualmente menos atractivo. Cada uno tiene, por lo tanto, sus objetivos, requerimientos y usuarios bien diferenciados.

\subsection{Viabilidad del modelo HBIM}

A la vista de las experiencias presentadas, podemos concluir que la creación del modelo HBIM es un aspecto crucial de cara a la viabilidad de su aplicación. En el campo de la obra nueva y la ingeniería, los presupuestos que se manejan son notablemente más altos que en el sector del patrimonio cultural, por lo que la repercusión del coste de la creación de un modelo BIM no es tan elevada. Además, las ventajas que se consiguen en la ejecución de la obra hacen más viable el uso de la metodología BIM. Sin embargo, en el ámbito del patrimonio, salvo excepciones, la cantidad de obra nueva ejecutada es escasa y los presupuestos más reducidos, por lo que el marco de viabilidad es más ajustado.

En concreto, entendemos que la aplicación de HBIM suele ser más provechosa en aquellos casos que impliquen el manejo de una elevada cantidad de información y/o cuando los datos han de interrelacionarse y deben ser accesibles. En cualquier caso, es fundamental planificar la metodología de modelado atendiendo al nivel de conocimiento del edificio, a los objetivos y a los usos planteados para poder establecer un LOD efectivo y, en última instancia, reducir los costes. Para ello, mientras no haya otros requisitos que exijan lo contrario, conviene simplificar el modelo al máximo, considerando el progresivo desarrollo del modelo en fases posteriores y en áreas determinadas según los requerimientos y recursos disponibles. De este modo, se debería abordar la coexistencia de varios modelos con distintos niveles de desarrollo vinculados entre sí, desde un modelo general del edificio con un alto nivel de simplificación hasta acercamientos en zonas concretas con un mayor nivel de desarrollo. Solo así se podrán obtener beneficios reales que puedan revertir a los promotores, a los usuarios y, en último término, a la sociedad.

\section{FICHA TÉCNICA Y AGRADECIMIENTOS}

La redacción de este trabajo se ha llevado a cabo en el marco del proyecto Tutela sostenible del patrimonio cul- 
tural a través de modelos digitales BIM y SIG. Contribución al conocimiento e innovación social. HAR201678113-R, financiado por MINECO-AEI/FEDER, UE. El estudio de la iglesia de Santiago de Peñalba fue encargado por la Dirección General de Patrimonio Cultura de la Junta de Castilla y León a Urbe pro Orbe Patrimonio Cultural, S.L. y a Control Bim, S.L. para su implementación en la Plataforma PetroBIMC. El estudio de San Pedro de la Mata se debe al proyecto Arqueología de la Arquitectura en la iglesia de San Pedro de la Mata (Sonseca, Toledo), cofinanciado por la Consejería de Educación, Cultura y Deportes, de la Junta de Comunidades de Castilla-La Mancha, el Instituto Geológico y Minero y el Ayuntamiento de Sonseca. El estudio de San Cebrián de Mazote se ha realizado en el marco del proyecto $\mathrm{Ar}$ queología de las iglesias hispánicas del siglo X: la circulación de los modelos arquitectónicos y decorativos. II. HAR2017-84927-P, financiado por MINECO-AEI/ FEDER, UE.

Todas las figuras reproducidas en este trabajo son propiedad de los autores. Agradecemos a los evaluadores sus indicaciones y aportaciones, cuya consideración ha enriquecido la redacción del texto final.

\section{BIBLIOGRAFÍA}

Banfi, F. 2017: "BIM orientation: grades of generation and information for different type of analysis and management process", The International Archives of the Photogrammetry, Remote Sensing and Spatial Information Sciences, XLII-2/W5, pp. 57-64.

Banfi, F., Brumana, R. y Stanga, C. 2019: "Extended reality and informative models for the architectural heritage: from scan-to-BIM process to virtual and augmented reality”, Virtual Archaeology Review, 10, pp. 14-30. https:// doi.org/10.4995/var.2019.11923

Bruno, N. y Roncella, R. 2018: "A restoration oriented HBIM system for cultural heritage documentation: the case study of Parma catedral", The International Archives of the Photogrammetry, Remote Sensing and Spatial Information Sciences, XLII-2, pp. 171-178.

Bruno, N. y Roncella, R. 2019: "HBIM for Conservation: A New Proposal for Information Modeling”, Remote Sensing, 11. https://doi.org/10.3390/ rs11151751

Caballero, L. 2010: "Experiencia metodológica en Arqueología de la Arquitectura de un grupo de investigación. Instituto de Historia. CSIC. Madrid", en C. Martín y E. de Vega (coords.), Arqueología aplicada al estudio e intervención de edificios históricos. Últimas tendencias metodológicas, pp. 103-120. Ministerio de Cultura, Madrid.

Castellano, M. y Pinto, F. 2019: "Dimensions and Levels of Knowledge in Heritage Building Information Modelling, HBIM: The model of the Charterhouse of Jerez (Cádiz, Spain)", Digital Applications in Archaeology and Cultural Heritage, 14. https://doi.org/10.1016/j.daach.2019.e00110

Guía de usuarios BIM, Documento 14. BIM aplicado al patrimonio cultural. Building SMART Spanish Chapter, Madrid. https:/www.buildingsmart.es/ recursos/gu\%C3\%ADas-ubim/

Jordan, I., Tzortzopoulos, P., García, J. y Pellicer, E. 2018: "Protocol to manage heritage-building interventions using heritage building information modelling (HBIM)", Sustainability, 10, 908. https://www.mdpi.com/2071$1050 / 10 / 4 / 908 / p d f$

Jouan, P. y Hallot, P. 2020: "Digital Twin: Research Framework to Support Preventive Conservation Policies", ISPRS International Journal of GeoInformation, vol 9, 228. https://doi.org/10.3390/ijgi9040228

Logothetis, S., Delinasiou, A. y Stylianidis, E. 2015: "Building information modelling for cultural heritage: a review", ISPRS Annals of the Photogrammetry, Remote Sensing and Spatial Information Sciences, II-5/W3, pp. 177-183.

López, F. J., Lerones, P. M., Llamas, J., Gómez, J. y Zalama, E. 2018: “A Review of Heritage Building Information Modeling (H-BIM)", Multimodal Technologies and Interact, 2, 21. http://dx.doi.org/10.3390/mti2020021

Martín, R., Cámara, L. y Murillo, J. I. 2018: “Análisis integrado de construcciones históricas: secuencia estratigráfica y diagnóstico patológico. Aplicación en la iglesia de Santa Clara (Córdoba)", Arqueología de la Arquitectura, 15, e067. https://doi.org/10.3989/arq.arqt.2018.001.

Martín, R., Murillo, J. I. y Utrero, M. a Á. 2020: "Key aspects regarding BIM for historical architecture. The church of San Cebrián de Mazote", en P. Ortiz, F. Pinto, P. Verhagen y A. J. Prieto (eds.) Science and Digital Technology for Cultural Heritage - Interdisciplinary Approach to Diagnosis, Vulnerability, Risk Assessment and Graphic Information Models, pp. 47-53. Taylor \& Francis Group, London. https://doi.org/10.1201/9780429345470

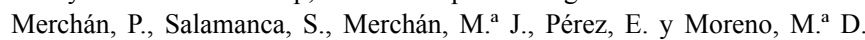
2018: "Pasado, presente y futuro de los HBIM (Heritage/Historic Building Information Models)", en Actas de las XXXIX Jornadas de Automática, pp. 1077-1084. Universidad de Extremadura, Badajoz. http://hdl.handle. net/10662/8884

Murphy, M., McGovern, E. y Pavia, S. 2009: "Historic building information modelling (HBIM)", Structural Survey, 27 (4), pp. 311-327. https://doi. org/10.1108/02630800910985108

Nieto, J. E. y Moyano J. J. 2014: "El estudio paramental en el modelo de información del edificio histórico o 'Proyecto HBIM'”, Virtual Archaeology Review, 5, pp. 73-85. https://doi.org/10.4995/var.2014.4183

Pocobelli, P., Bohem, J., Bryan, P., Still, J. y Grau-Bové, J. 2018: “BIM for heritage science: a review", Heritage Science, 6, 30. https://doi.org/10.1186/ s40494-018-0191-4

Salvador, E., García-Valldecabres, J. y Viñals, M. J. 2018: "The use of HBIM models as a tool for dissemination and public use management of historical architecture: a review", International Journal of Sustainable Development and Planning, 13, pp. 96-107. https://doi.org/10.2495/SDPV13-N1-96-107

The American Institute of Architects. 2013: AIA G202 ${ }^{\text {TM}}-2013$ Project Building Information Modelling Protocol Form. http://content.aia.org/ sites/default/files/2016-09/AIA-G202-2013-Free-Sample-Preview.pdf 